\title{
Gradient Methods in the Solution of Systems of Linear Equations
}

\author{
Marvin L. Stein
}

\begin{abstract}
The results of various experiments with iterative methods for solving systems of linear algebraic equations are discussed. Modifications of the optimum gradient method are compared, and the rather interesting self-acceleration properties of a class of methods here named "almost optimum" gradient methods are pointed out.
\end{abstract}

\section{Introduction}

The method of steepest descent, or the optimum gradient method, has been known to mathematicians since the time of Cauchy [1]. ${ }^{2}$ Others who have discussed this method include Curry |2], Forsythe and Motzkin [3], Householder [4], Kantorovitch [5], and Temple [6]. Its infrequent application in computationa! work is no doubt due to the slowness with which it converges. This slowness of convergence is unfortunately generally true of gradient methods. However, with the advent of large-scale computing machinery it has become feasible to seriouslv consider them in practical numerical analvsis.

In a forthcoming paper, Hestenes and Stein [7] discuss a large class of gradient procedures for solving systems of linear equations. These procedures contain the optimum gradient method as a special case. The present note is mainly a report on some numerical exneriments with them that were carried out on the IBM Card-Programmed Electronic Calculator at the Institute for Numerical Analysis of the National Bureau of Standards. Some attention is also given to an experiment in which the problem of solving a system of linear equations was changed to an equivalent eigenvalue problem and then solved by a modification of one of the gradient methods riscussed by Hestenes and Karush [8]. The most striking result of the experiments indicates that there is a large class of gradient methods that is self-accelerating, that is, which irregularly shows a large increase in the rate of convergence without the introduction of any modi. fication in the computational routine. This behavior is in sharp contrast to that of the method of steepest descent, which in the light of the present results can no longer be considered as optimum from an over-all point of view unless modified by some special accelerating routine [9].

\section{Summary of the Theory}

Let $A$ denote an $m$-rowed and $n$-columned matrix, and let $b$ and $x$ be $m$-rowed and $n$-rowed column vectors, respectively. The vector $r(x)=b-A x$ is then an $m$-rowed column vector. In the following a $\operatorname{star}(*)$ affixed to the symbol for any vector or matrix will indicate its conjugate transpose. Hence.

\footnotetext{
1 The preparation of this paper was sponsored (in part) by the Office of Naval Research.

${ }_{2}^{2}$ Figures in brackets indicate the literature references at the end of this paper.
}

if $H$ is a positive $m \times m$ Hermitian matrix, we have $H^{*}=H, \quad r * H r>0$, provided $r \neq 0$. Clearly, the nonnegative real-valued function

$$
f(x)=r^{*} H r
$$

vanishes if and only if

$$
A x=b \text {. }
$$

Therefore, the problem of minimizing (1) is equivalent to the problem of solving the svstem (2), provided a solution exists. If no solution exists, a vector $x$ which minimizes $f(x)$ vields a best fit of $b$ by $A x$ in a least-squares sense with the metric determined bv $H$.

Hestenes and Stein [7] have analyzed the following algorithm for constructing the minimum of (1). Consider iterations of the type

$$
x_{i+1}=x_{i}+\alpha_{i} \xi_{i} \quad(i=0,1,2, \ldots),
$$

where $x_{0}$ is chosen initially and where, after $x_{i}$ has been determined, the gradient vector $\xi_{i}$ is defined by the rule $\xi_{i}=A^{*} \operatorname{Ir}\left(x_{i}\right)$. If $\xi_{i}=0$, the problem is solved. If $\xi_{i} \neq 0$, the scalar $\alpha_{i}$ is taken to be of the form $\alpha_{i}=\beta_{i} \gamma_{i}$, where

$$
\gamma_{i}=\frac{\xi_{i}^{*} \xi_{i}}{\xi_{i}^{*} A^{*} H A \xi_{i}}
$$

and $\beta_{i}$ is any complex number. The sequence (3) has been shown to converge to the minimum of $f(x)$ provided the coefficients $\beta_{i}$ satisfy the conditions

$$
\frac{1}{\beta_{i}}+\frac{1}{\bar{\beta}_{i}} \geq 1+\delta, \quad\left|\beta_{i}\right| \geq \delta,
$$

where $\delta$ is arbitrary on the range $0<\delta<1$.

Setting $\beta_{i} \equiv 1$ gives the optimum gradient method, which has the following geometrical interpretation. Starting at $x_{0}$, one proceeds along the normal to that member of the family of concentric ellipsoids

$$
f(x)=\text { constant },
$$

which passes through $x_{0}$ until a point tangent to another ellipscid of the family is reached. One then goes along the normal to this second ellipsoid until one is again at a point of tangency to a member of (4), 
and so on until the common center is reached. Clearly, it would be highly desirable to land on an axis of the family. However, as simple two-dimensional examples illustrate, one usually overshoots the major axis by proceeding all the way to a point of tangency. Hence, it was conjectured by M. R. Hestenes that of the allowed values of $\beta_{i}$ some of those chosen from the range $\beta_{i}<1$ would yield better convergence than $\beta_{i} \equiv 1$. This conjecture is the motivation for the experimental work whose results will be tabulated and discussed below.

\section{Experimental Data}

The algorithm described in the preceding section was carried out for the problem $A x=b$ with

$$
A=\left(\begin{array}{rrrrrr}
.06667 & .02634 & -.04640 & -.07368 & -.02131 & -.00431 \\
.02634 & .26841 & -.02243 & .15952 & -.05923 & -.12797 \\
-.04640 & -.02243 & .10932 & .05150 & -.04100 & .08558 \\
-.07368 & .15952 & .05150 & .25152 & -.01141 & -.07169 \\
-.02131 & -.05923 & -.04100 & -.01141 & .14403 & .01105 \\
-.00431 & -.12797 & .08558 & -.07169 & .01105 & .19450
\end{array}\right)
$$

and

$$
b=\left(\begin{array}{r}
-.008609 \\
-.014279 \\
-.000243 \\
.004576 \\
.008043 \\
-.004895
\end{array}\right) \text {. }
$$

Runs were made with fixed values of $\beta_{i}$ ranging between $\beta_{i} \equiv .1$ and $\beta_{i} \equiv 1.9$. Since the matrix $A$ and the vector $b$ were obtained from an original matrix $B$ and vector $c$ as

$$
A=B^{*} B, \quad b=B^{*} c,
$$

$A$ is positive and symmetric. Hence, a convenient metric is

$$
H=A^{-1} .
$$

In the metric (8) the gradient becomes $\xi_{i}=r\left(x_{i}\right)$. Thus the other significant quantities defined in section 2 assume the form

$$
\begin{aligned}
\gamma_{i} & =\frac{r^{*}\left(x_{i}\right) r\left(x_{i}\right)}{r^{*}\left(x_{i}\right) A r\left(x_{i}\right)} \\
f\left(x_{i}\right) & =c^{*} c-\left(x_{i}^{*} b+b^{*} x_{i}\right)+x_{i}^{*} A x_{i} \\
& =c^{*} c-x_{i}^{*}\left(b+r\left(x_{i}\right)\right) .
\end{aligned}
$$

Table 1 lists runs of $f\left(x_{i}\right)$ and $f\left(x_{i}\right) / f\left(x_{i-1}\right)$ for various fixed values of $\beta_{i}$. In each case the initial $x=x_{0}$ was zero. The values of $f\left(x_{i}\right)$ are given here to the same number of places as in the original calculation, while the ratios $f\left(x_{i}\right) / f\left(x_{i-1}\right)$ have been cut down from a six-place table. Table 2 gives the values of $\alpha_{i}$ corresponding to the runs listed in table 1. These numbers were originally computed to 10 places. The results listed in tables 1 and 2 are based for the most part on single runs of the Card-Programmed Calculator. The main check used in the machine computations was the monotonicity of $f(x)$. However, the run $\beta_{i} \equiv .9$ has been exactly duplicated on two separate occasions The results of the run $\beta_{i} \equiv 1$ check closely with results obtained by $\mathrm{A}$. I. Forsythe in an entirely independent manner. 
Table 1

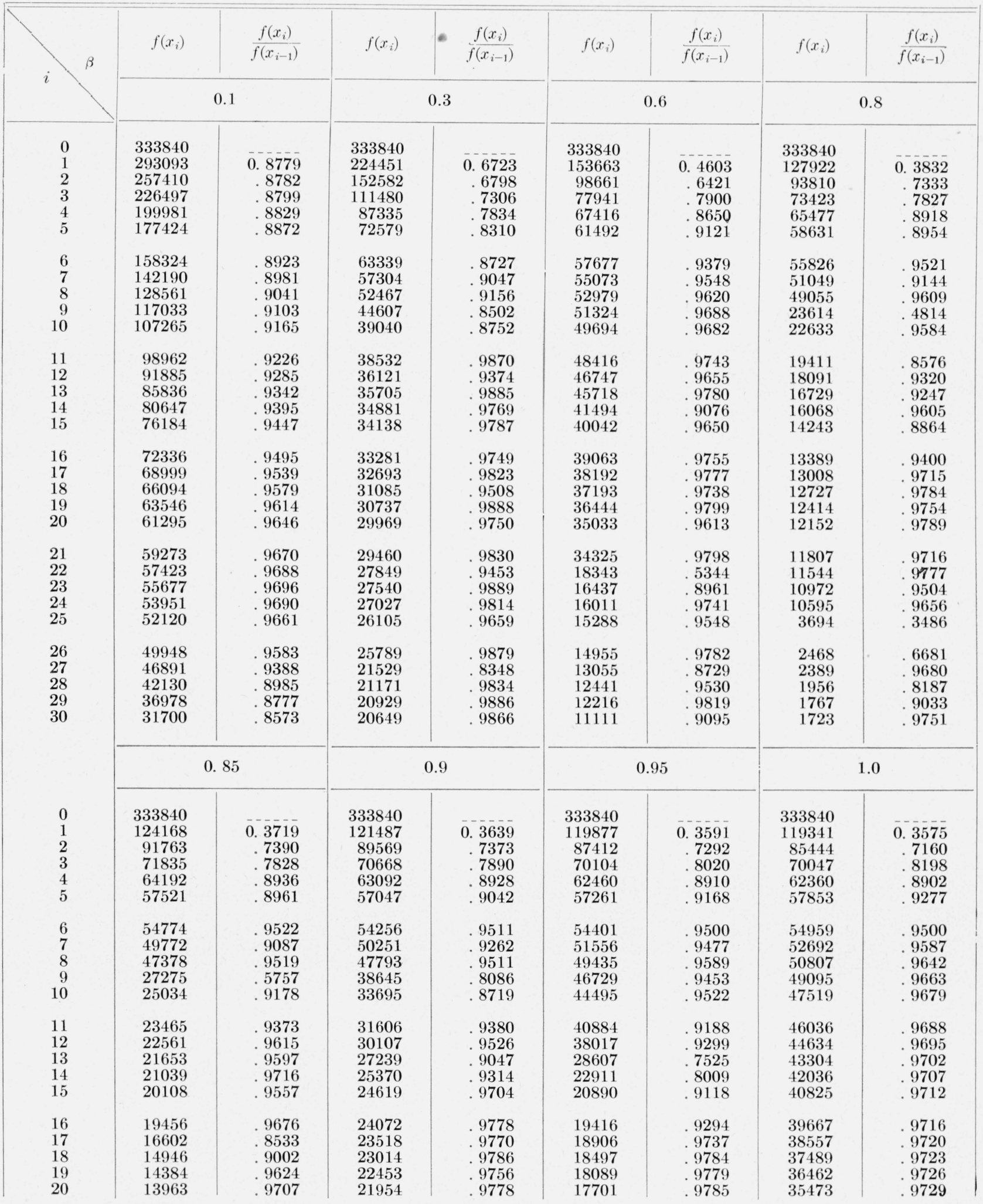


TABLE 1.-Continued

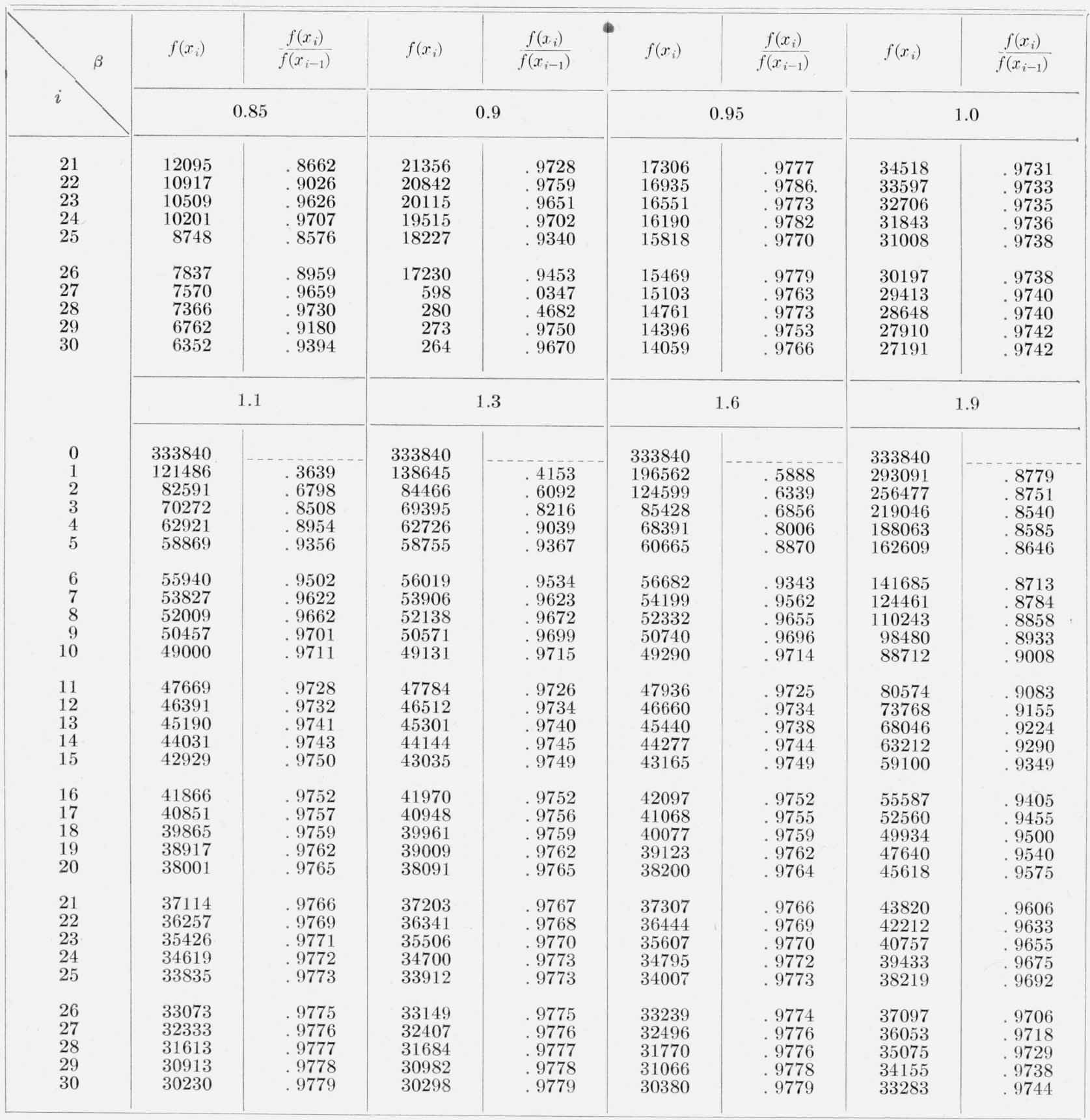


TABLE 2 .

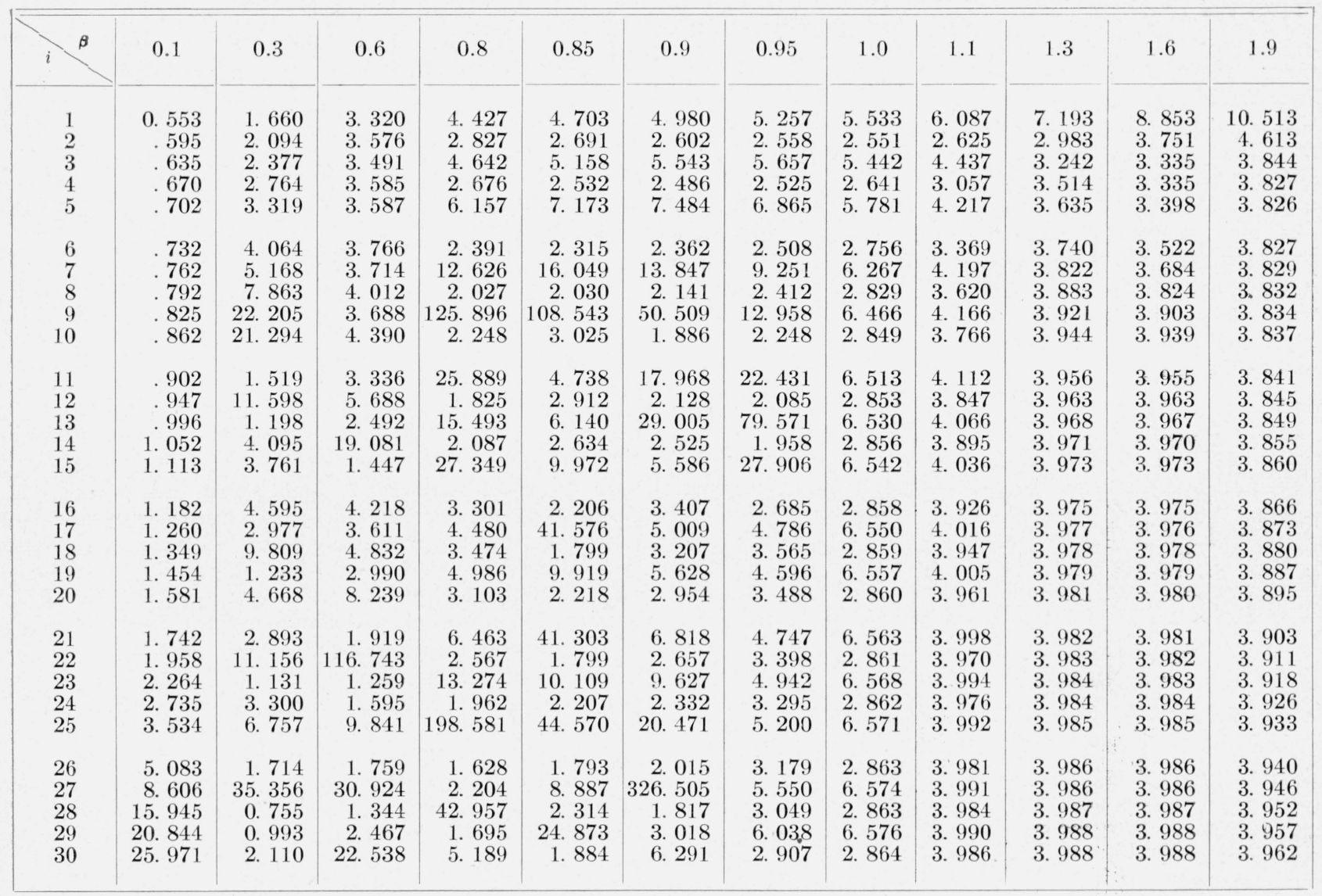

\section{Conversion to an Eigenvalue Problem}

By introducing the variable $x_{n+1}$, an equation $B x=c$ can be expressed in the homogeneous form $C y=0$, where

$$
C=\left(\begin{array}{ll}
B & c
\end{array}\right), \quad y=\left(\begin{array}{l}
x \\
x_{n+1}
\end{array}\right) .
$$

Multiplying through by $C^{*}$ gives the system of order one higher $D y=0$, where

$$
D=\left(\begin{array}{ll}
B^{*} B & B^{*} c \\
c^{*} B & c^{*} c
\end{array}\right)
$$

is a symmetric matrix whose lesst eigenvalue is zero. The nontrivial eigenvector corresponding to this zero eigenvalue yields a solution of the original system of linear equations.

As is well known, the least eigenvector of the symmetric matrix $D$ can be found by minimizing the Rayleigh quotient

$$
\mu(y)=\frac{y^{*} D y}{y^{*} y}, \quad y \neq 0 .
$$

Hestenes and Karush [8] have examined in detail the convergence of various methods for accomplishing the minimization of $\mu$. The following algorithm for constructing a sequence $\left\{y_{i}\right\}$ that minimizes $\mu$ is a modification of their "optimum $\alpha$ " procedure. We define the gradient as $\xi_{i} \equiv \xi\left(y_{i}\right)=D y_{i}-\mu\left(y_{i}\right) y_{i}$. Let

$$
\gamma_{i}=\frac{1}{\mu\left(\xi_{i}\right)}, \quad \mu\left(\xi_{i}\right) \neq 0,
$$

and let $\alpha_{i}=\beta \gamma_{i}$, where $\beta$ is a positive number less than or equal to one. Then, given $y_{i}$, we determine $y_{i+1}$ by the formula $y_{i+1}=y_{i}-\alpha_{i} \xi_{i}$.

This algorithm was actually carried out with the matrix

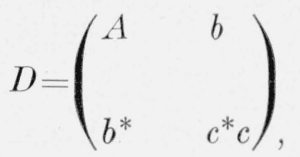

where $A$ is given by (5), $b$ is given by (6) with all components multiplied by 10 and $c^{*} c=.333840$. In view of (7) $D y=0$ is actually a problem of the type just described. Runs were made with various values of $\beta$ ranging between .7 and 1. For purposes of comparison, the same starting point (origin) as 
in the computations recorded in section 3 was used and a record of

$$
f(x)=\frac{y^{*} D y}{\left|y_{n+1}\right|^{2}}
$$

was kept. In table $3^{3}$ we present the results of the run $\beta=.9$. The runs for other values of $\beta$ showed substantially the same unstable behavior, while that for $\beta=1$ (optimum) showed a very stable behavior and as a result converged quite slowly. The numbers $f\left(x_{i}\right)$ and $\alpha_{i}$, appearing in table 3 , were originally computed to 10 places. The ratios $f\left(x_{i}\right) / f\left(x_{i-1}\right)$ were computed from the original values of $f\left(x_{i}\right)$ and then cut down to the present size.

TABLE 3 .

\begin{tabular}{|c|c|c|c|}
\hline$i$ & $f\left(x_{i}\right)$ & $f\left(x_{i}\right) / f\left(x_{i-1}\right)$ & $0.9 \gamma_{i}$ \\
\hline 0 & 333840 & & \\
\hline 1 & 121482 & 0.3639 & 0.4980 \\
\hline 2 & 86700 & .7137 & 2575 \\
\hline 3 & 70323 & .8111 & .7076 \\
\hline 4 & 57533 & .8181 & .3951 \\
\hline 5 & 60698 & 1. 0550 & .5478 \\
\hline 6 & 53564 & 0.8825 & . 3968 \\
\hline 7 & 53869 & 1. 0057 & 5494 \\
\hline 8 & 49788 & 0.9242 & 3479 \\
\hline 9 & 46902 & .9420 & .5237 \\
\hline 10 & 45704 & .9744 & .3046 \\
\hline 11 & 41266 & 9029 & 5577 \\
\hline 12 & 41045 & .9947 & 2761 \\
\hline 13 & 36337 & .8853 & 6639 \\
\hline 14 & 36011 & .9910 & 2482 \\
\hline 15 & 31318 & .8697 & . 9109 \\
\hline 16 & 30291 & .9672 & . 2189 \\
\hline 17 & 24919 & .8226 & 1. 7145 \\
\hline 18 & 22095 & .8867 & 0. 1909 \\
\hline 19 & 7468 & . 3380 & 5. 9172 \\
\hline 20 & 6963 & .9324 & 0. 2758 \\
\hline 21 & 5985 & .8595 & 6081 \\
\hline 22 & 5541 & 9258 & 2493 \\
\hline 23 & 4642 & 8378 & 8282 \\
\hline 24 & 4206 & 9060 & .2201 \\
\hline 25 & 3171 & .7540 & 1. 5481 \\
\hline 26 & 2644 & 8339 & 0. 1912 \\
\hline 27 & 264 & 0998 & 6. 6970 \\
\hline 28 & 151 & 5733 & 0. 1766 \\
\hline 29 & 117 & .7708 & 1. 4380 \\
\hline 30 & 91 & .7806 & 0. 1908 \\
\hline 31 & 11 & 1274 & 6. 4755 \\
\hline 32 & 8 & . 6933 & 0. 1890 \\
\hline
\end{tabular}

\section{Conclusion}

The error function $f(x)$ goes monotonically to zero in each of the gradient methods. Hence the number $\mathrm{P}_{n}=100 f\left(x_{n}\right) / f\left(x_{0}\right)$ tells us what percentage of the distance from the starting point to zero remains to be covered at the $n$th step. Table 4 gives $P_{30}$ for various values of $\beta_{i}$. We note

3 The author thanks R. M. Hayes for furnishing him with most of the data appearing in table 3.
TABLE 4 .

\begin{tabular}{|c|c|c|c|c|c|c|}
\hline$\beta$ & 0.1 & 0.3 & 0.6 & 0.8 & 0.85 & 0.9 \\
$P_{30}$ & 9.49 & 6.18 & 3.33 & 0.52 & 1.90 & 0.08 \\
\hline \hline$\beta$ & 0.95 & 1.0 & 1.1 & 1.3 & 1.6 & 1.9 \\
$P_{30}$ & 4.21 & 8.14 & 9.05 & 9.07 & 9.10 & 9.97 \\
\hline
\end{tabular}

after 30 steps that, with the exception of the case $\beta_{i} \equiv \beta=.1$, all the gradient methods for values $\beta<1$ are converging faster than the optimum method, while all the gradient methods for values $\beta>1$ are converging at a slightly slower rate than the optimum method. For the eigenvalue method we have $P_{32}=.002$. The increased rate of convergence for this case is offset on the Card-Programmed Calculator by the greater length of time needed for each step. However, on a high-speed machine this factor would be negligible. An explanation of the speedier convergence of the eigenvalue procedure lies in the fact that the transformation of the problem to the homogeneous form has shrunk the ratio of the largest and the smallest nonzero eigenvalues. This improvement of "condition" is something that one could not generally expect to occur [10].

The ratios $f\left(x_{i}\right) / f\left(x_{i-1}\right)$ compare the rate of convergence at each step with that of a geometric progression having the same ratio. A study of these numbers and of the corrections $\alpha_{i}$ brings into sharp focus the contrast between the instability of the gradient methods employing $\beta<1$ and the stability of those employing $\beta \geq 1$. In the method of steepest descent it is just this stability that permits acceleration. However, this acceleration must be achieved through a modification of the computational routine. On the other hand, the instability of the methods using $\beta<1$ leads to occasional accelerations without the introduction of any changes whatsoever in the computing routine.

From the point of view of using the "almost optimum" gradient method on a large scale computer, its self-acceleration property has more than theoretical interest. As is well known, the high-speed memory capacity of the computers now in existence is rather limited. Hence, the necessity of storing a special acceleration routine might prove to be a severe handicap indeed.

It is worth while to compare the values of $\alpha_{i}$ with the reciprocal eigenvalues of the matrices $A$ and $D$. For $A$ these reciprocal eigenvalues have been found to be approximately $2.0,3.9,5.7,12.1,63$, and 372 , while for $D$ the finite reciprocal eigenvalues range between approximately 8.35 and $0.189 .^{4}$ We will pay particular attention to the points at which acceleration took place. One sees that preceding an acceleration there was a "smoothing run" during which the $\alpha_{i}$ 's were in the range of the small reciprocal eigenvalues. On the iteration immediately before an acceleration, $\alpha_{i}$ was almost equal to the smallest reciprocal eigenvalue, while on the iteration

\footnotetext{
${ }_{4}$ These values were furnished by R. M. Hayes.
} 
during which acceleration took place $\alpha_{i}$ was between the highest and next to highest reciprocal eigenvalues. It was just this technique of choosing $\alpha_{i}$ that the present author helped develop in previous experiments with a "fixed $\alpha$ " gradient method, which were conducted under the direction of M. R. Hestenes. In this method the operator chooses the value of $\alpha$ just before each iteration, and by judicious choices he can successfully accelerate the method to a considerable extent. However, this requires too many judgments of the operator to be practical for a fast machine or an inexperienced operator. Hence, it is quite hopeful to note the existence of methods suitable for high-speed machines that can duplicate the fixed $\alpha$ acceleration procedures without any intervention by the operator once the process has started.

\section{References}

[1] A. Cauchy, Méthode générale pour la résolution des systèmes d'équations simultanées, Compt. rend. L'Académie Sciences 25, 536 (1847).

[2] H. B. Curry, The method of steepest descent for nonlinear minimization problems, Quart. Applied Math. 2, 258 (1944).

[3] G. E. Forsythe and T. S. Motzkin, Asymptotic properties of the optimum gradient method, Bul. Am. Math. Soc. 5\%, 183 (1951).
[4] A. S. Householder, Notes on numerical methods (1949 51) (typescript available at Oak Ridge National Laboratory).

[5] L. V. Kantorovitch, On an effective method of solving extremal problems for quadratic functionals, Compt. rend. (Doklady) de l'Académie des Sciences de l'URSS 48, 455 (1945).

[6] G. Temple, The general theory of relaxation methods applied to linear systems, Proc. Roy. Soc. (London) $\mathbf{1 6 9}$ [A] 476 (1938-39).

[7] M. R. Hestenes and M. L. Stein, The solution of linear equations by minimization, in manuscript at the National Bureau of Standards, Los Angeles, Calif.

[8] M. R. Hestenes and W. Karush, A method of gradients for the calculation of the characteristic roots and vectors of a real symmetric matrix, J. Research NBS 47, 45 (1951) RP2227.

[9] The problem of accelerating the optimum gradient method has recently been considered by G. E. Forsythe and T. S. Motzkin in a paper entitled, Acceleration of the optimum gradient method, presented to the American Mathematical Society (Stanford meeting) (April 28,1951 ).

[10] For a discussion of the effect of this type of transformation on the condition of a matrix, see G. E. Forsythe and T. S. Motzkin, An extension of Gauss's transformation for improving the condition of systems of linear equations, typescript available at National Bureau of Standards, Los Angeles, Calif.

Los Angeles, August 13, 1951. 